\section{HIGH-POTENCY SYNTHETIC STING AGONISTS REWIRE THE MYELOID STROMA IN THE TUMOUR MICROENVIRONMENT TO AMPLIFY IMMUNE CHECKPOINT BLOCKADE EFFICACY IN REFRACTORY PANCREATIC DUCTAL ADENOCARCINOMA}

${ }^{1}$ Akash Boda*, ${ }^{2}$ Casey Ager, ${ }^{3}$ Kimal Rajapakshe, ${ }^{1}$ Spencer Lea, ${ }^{4}$ Maria Emilia Di Francesco, ${ }^{4}$ Philip Jones, ${ }^{4}$ Michael Curran. ${ }^{1}$ University of Texas MD Anderson UTHealth Graduate School of Biomedical Sciences, Houston, TX, United States; ${ }^{2}$ Columbia University Irving Medical Center, New York, NY, United States; ${ }^{3}$ Baylor College of Medicine, Houston, TX, United States; ${ }^{4}$ UT MD Anderson Cancer Center, Houston, TX, United States

Background Pancreatic ductal adenocarcinoma (PDAC) is one of the most lethal malignancies and is clinically unresponsive to immune checkpoint blockade (ICB) immunotherapy. ${ }^{1}{ }^{2}$ High densities of immunosuppressive myeloid cells, ${ }^{3}$ a paucity of antigen-presenting cells ${ }^{4-6}$ and $\mathrm{T}$ cell exclusion from tumour microenvironment ${ }^{7}$ all contribute to the refractory nature of PDAC to immune-based therapies. We and others have shown that innate immune activation of myeloid stroma via engagement of the STING (Stimulator of Interferon Genes) pathway can mediate proinflammatory remodeling and trigger a flood of $\mathrm{T}$ cell infiltration into otherwise 'cold' tumours. ${ }^{8-11}$ To that end, intratumoral injection of cyclic dinucleotide (CDN) agonists of the STING pathway has been shown to foster local and abscopal tumor immunity. ${ }^{8-10}$ Despite proven therapeutic efficacy in preclinical models, the mechanistic basis at a cellular level of how CDNs reprogram the suppressive myeloid stroma to sensitise tumours to ICB is poorly understood.

Methods Using RNA sequencing and protein arrays we profiled myeloid-derived suppressor cell (MDSC) and M2 macrophage function following stimulation with CDNs of ascending potency. We describe the effects of CDN STING agonists on cell cycle dynamics, metabolic reprogramming and c-Myc expression in MDSCs. Next, in an orthotopic Kras+/ G12DTP53+/R172HPdx1-Cre (KPC)-derived model of PDAC, we determined the ability of intratumorally-administered CDNs to sensitise PDAC to checkpoint blockade using bioluminescent in vivo imaging and multi-parameter flow cytometry of tumor stroma post-therapy.

Results Multi-omics profiling of MDSCs and M2 Macrophages of human and murine origin show that high-potency synthetic STING agonists rewire these populations from immunosuppressive to immune-permissive phenotypes in part through inhibition of c-Myc signaling, energy metabolic modulation, and antagonism of cell cycle. Intratumoral injection of the STING agonist, IACS-8803 resulted in an amplified therapeutic response to checkpoint blockade that was dependent on $\mathrm{T} /$ NK cell infiltration into the tumour. Furthermore, dimensionality reduction analyses of multiparameter flow cytometry data show proinflammatory remodeling of the myeloid stroma and enhanced $\mathrm{T}$ cell function as salient features of synthetic agonists versus natural CDNs in orchestrating the in vivo therapeutic benefit.

Conclusions This study uncovers molecular and cellular mechanisms by which STING agonists drive proinflammatory conversion of tumour myeloid stroma. We are the first to report that synthetic CDN STING agonists affect MDSC and M2 macrophage repolarization through altering energy metabolism and c-Myc signalling. Lastly, we demonstrate the potential for high-potency STING agonists to overcome resistance to checkpoint blockade in an aggressive orthotopic tumour model of PDAC.

\section{REFERENCES}

1. Royal RE, Levy $C$, et al. Phase 2 trial of single agent Ipilimumab (anti-CTLA-4) for locally advanced or metastatic pancreatic adenocarcinoma. J Immunother 2010;33 (8):828-33.

2. Brahmer JR, Tykodi SS, et al. Safety and activity of anti-PD-L1 antibody in patients with advanced cancer. N Eng/ J Med 2012;366(26):2455-65.

3. Karakhanova S, Link J. Characterization of myeloid leukocytes and soluble mediators in pancreatic cancer: importance of myeloid-derived suppressor cells. Oncoimmunology 2015:4:e998519.

4. Dallal RM, Christakos P, et al. Paucity of dendritic cells in pancreatic cancer. Surgery 2002;131:135-138

5. Yamamoto $\mathrm{T}$, Yanagimoto $\mathrm{H}$, et al. Circulating myeloid dendritic cells as prognostic factors in patients with pancreatic cancer who have undergone surgical resection. I Surg Res 2012;173:299-308.

6. Hegde $S$, Krisnawan $V$, et al. Dendritic cell paucity leads to dysfunctional immune surveillance in pancreatic cancer. Cancer Cell 2020;37(3):289-307.

7. Beatty GL, Winograd R, et al. Exclusion of $T$ cells from pancreatic carcinomas in mice is regulated by Ly6Clow F4/80+ extratumoral macrophages. Gastroenterology 2015;149(1):201-210

8. Baird JR, Friedman D, et al. Radiotherapy combined with novel STING-Targeting oligonucleotides results in regression of established tumors. Cancer Res 2016;76 (1):50-61.

9. Ager CR, Reilley MJ, et al. Intratumoral STING activation with T-cell checkpoint modulation generates systemic antitumor immunity. Cancer Immunol Res 2017:5 (8):676-84.

10. Smith TT, Moffett $H F$, et al. Biopolymers codelivering engineered $T$ cells and STING agonists can eliminate heterogeneous tumors. J Clin Invest 2017;127 (6):2176-91.

11. Jing W, McAllister D, et al. STING agonist inflames the pancreatic cancer immune microenvironment and reduces tumor burden in mouse models. I Immunother Cancer 2019;7(1):115.

http://dx.doi.org/10.1136/jitc-2021-SITC2021.758 